Article

\title{
Improving DGNSS Performance through the Use of Network RTK Corrections
}

\author{
Duojie Weng ${ }^{1,2}$, Shengyue $\mathrm{Ji}^{3}$, Yangwei $\mathrm{Lu}^{1,2, *}$, Wu Chen ${ }^{2} \mathbb{D}$ and Zhihua $\mathrm{Li}^{4}$ \\ 1 Shenzhen Research Institute, The Hong Kong Polytechnic University, Shenzhen 518063, China; \\ ceweng@polyu.edu.hk \\ 2 Department of Land Surveying and Geo-Informatics, Hong Kong Polytechnic University, Hong Kong, China; \\ wu.chen@polyu.edu.hk \\ 3 College of Oceanography and Space Informatics, China University of Petroleum (East China), \\ Qingdao 266580, China; 19990045@upc.edu.cn \\ 4 College of Energy and Electrical Engineering, Hohai University, Nanjing 211100, China; zhli@hhu.edu.cn \\ * Correspondence: lu.y.lu@polyu.edu.hk
}

Citation: Weng, D.; Ji, S.; Lu, Y.; Chen, W.; Li, Z. Improving DGNSS Performance through the Use of Network RTK Corrections. Remote Sens. 2021, 13, 1621. https://doi.org/ $10.3390 /$ rs13091621

Academic Editor: Xiaolin Meng

Received: 24 March 2021

Accepted: 19 April 2021

Published: 21 April 2021

Publisher's Note: MDPI stays neutral with regard to jurisdictional claims in published maps and institutional affiliations.

Copyright: (c) 2021 by the authors. Licensee MDPI, Basel, Switzerland. This article is an open access article distributed under the terms and conditions of the Creative Commons Attribution (CC BY) license (https:// creativecommons.org/licenses/by/ $4.0 /)$.

\begin{abstract}
The differential global navigation satellite system (DGNSS) is an enhancement system that is widely used to improve the accuracy of single-frequency receivers. However, distancedependent errors are not considered in conventional DGNSS, and DGNSS accuracy decreases when baseline length increases. In network real-time kinematic (RTK) positioning, distance-dependent errors are accurately modelled to enable ambiguity resolution on the user side, and standard Radio Technical Commission for Maritime Services (RTCM) formats have also been developed to describe the spatial characteristics of distance-dependent errors. However, the network RTK service was mainly developed for carrier-phase measurements on professional user receivers. The purpose of this study was to modify the local-area DGNSS through the use of network RTK corrections. Distancedependent errors can be reduced, and accuracy for a longer baseline length can be improved. The results in the low-latitude areas showed that the accuracy of the modified DGNSS could be improved by more than $50 \%$ for a $17.9 \mathrm{~km}$ baseline during solar active years. The method in this paper extends the use of available network RTK corrections with high accuracy to normal local-area DGNSS applications.
\end{abstract}

Keywords: DGNSS; network RTK; accuracy; distance-dependent errors

\section{Introduction}

The global navigation satellite system (GNSS) suffers from various errors due to the ionosphere, troposphere, and satellite orbit and clock. These errors can be significantly reduced with the application of differential GNSS (DGNSS) corrections generated from a nearby reference station at a known location. Since the DGNSS is easy to use, it is supported by most low-cost GNSS receivers. Today, raw measurements are available from smartphones, and DGNSS is a basic technique that improves the accuracy of smartphone positioning [1]. The accuracy of DGNSS is mainly affected by uncommon errors and decorrelation errors. First, receiver noise and multipath effects on the user side are not common with these at the reference station; hence, these factors determine the accuracy level of DGNSS. Aside from uncommon errors, temporal and spatial decorrelation errors also affect DGNSS performance.

Temporal decorrelation errors are caused by the latency between the generation of corrections and their application. A reference station provides a pseudorange correction (PRC) for each satellite at each epoch, and a user may apply the correction a few seconds later due to transmission delay. In this case, PRC loses its validity when the user applies it, resulting in the temporal decorrelation error. Range rate correction (RRC) is thus estimated and provided to users, enabling them to overcome the temporal decorrelation error. When 
selective availability (SA) was in effect, errors in the fundamental frequency of the GPS clocks can be as large as $1000 \mathrm{~m}$, and latency compensation could significantly improve differential global positioning system (DGPS) performance. Since SA was turned off in 2000, the GPS error became much smaller, and temporal decorrelation was reduced to a negligible value [2]. On the other hand, distance-dependent errors are caused by the spatial decorrelation of orbit and the propagation errors [3,4]. Today, distance-dependent errors are the largest error sources in DGNSS, and they receive extensive attention, especially for applications that require higher accuracy and integrity [5,6].

Distance-dependent errors affect integrity performance, which is important for safetycritical applications, such as aircraft approaching and landing. Some can be detected by the code carrier divergence (CCD) monitor [7]. Others go undetected, which poses a potential threat to the integrity of DGNSS. Extensive research efforts were thus made to develop a threat model to protect against undetected distance-dependent errors $[5,8]$. The combination of the CCD monitor and the threat model was used in the mid-latitude region. In low-latitude regions, large ionospheric variability frequently happens, and the availability of the system can be degraded if the threat model is used $[9,10]$.

In the past two decades, the satellite-based augmentation systems (SBASs) were developed to overcome distance-dependent errors in a wide area. SBASs collect GPS data from a network of reference stations installed across a wide area and models for various distance-dependent errors. These model parameters are relayed to users via geostationary satellites. For example, vertical ionospheric delay at a number of grid points is generated and provided to users, which enables them to interpolate the ionospheric correction at their own positions. An SBAS efficiently extends the coverage of DGPS to a wide area. This was globally developed, including the wide-area augmentation system (WAAS) in the United States, the European geostationary navigation overlay service (EGNOS) in Europe, and GPS-aided GEO augmented navigation (GAGAN) in India.

In a local area, distance-dependent errors can also be reduced by using pseudorange corrections from multiple reference stations [11-13]. In this method, pseudorange corrections from multiple reference stations are combined in the user receiver, and the optimal correction is usually interpolated at the user location. In [4], the satellite-elevation-based interpolation model was better than the linear interpolation model, particularly when the user was outside of the network. In local-area DGNSS with multiple reference stations, pseudorange corrections from multiple reference stations contain distance-independent errors, such as multipath effects on code measurements for individual reference stations. Distance-independent errors, however, do not fit the interpolation model.

Another method is to model distance-dependent errors using multiple reference stations. The correction that accounts for distance-related errors can then be added to the pseudorange correction generated from the single station. The correction can be obtained from various ionospheric models such as the grid model, but modeling the ionosphere is challenging, especially during the period of ionospheric anomalies [14,15]. In our previous study, the ionospheric gradient model was developed to reduce differential ionospheric errors, thereby improving DGNSS performance [9]. In this model, the spatial variability of ionospheric delay for each satellite is described using the east-west and north-south coefficients, which are generated from dual-frequency carrier-phase observables of more than three reference stations.

The continuous operational reference system (CORS) network was extensively globally established to enable accurate positioning through the real-time kinematic (RTK) technology in the 1990s [16]. RTK obtains double-differenced phase measurements and resolves double-differenced ambiguities to integer values. In order to correctly resolve integer ambiguities, the rover needs to be close enough to the reference station due to distance-dependent errors in double-differenced phase observables [17]. To extend baseline length, the network RTK was developed to model distance-dependent errors using multiple reference stations in a region. Among different distance-dependent errors, the ionospheric effect is much larger than other errors are, even in mid-latitude regions under 
calm ionospheric conditions [18]. Thus, extensive researcher efforts have aimed at improving the accuracy of the differential model of the ionosphere $[11,19,20]$. Today, network RTK technology is implemented by correction techniques, e.g., virtual reference stations (VRSs), area-correction parameters (KFP), and the master auxiliary concept (MAC) [21]. These techniques accurately model distance-dependent errors and describe the spatial characteristics of distance-dependent errors of the ionosphere, ionosphere, and orbits.

In many global regions, a network RTK service with high-precision accuracy is readily available to support various applications. However, the network RTK was mainly developed for the carrier-phase measurements of professional receivers. Low-cost receivers that only support code-based positioning could not benefit from accurate corrections of network RTK; thus, the performance of a conventional DGNSS was limited. The purpose of this study is to propose a modified DGNSS that improves positioning performance through the use of network RTK corrections without modifying the hardware on the user side. Specifically, accurate corrections for distance-dependent errors in network RTK were incorporated into the DGNSS. If network RTK corrections can work in DGNSS, the positioning performance of the code-based DGNSS can be significantly improved, and the network RTK service can be extended to normal DGNSS receivers. The proposed DGNSS in this work can be used in areas where a network RTK service is available.

\section{Distance-Dependent Errors in Traditional DGNSS and Network RTK}

Distance-dependent errors in conventional DGNSS are first illustrated. Then, how distance-dependent errors are mitigated in the network RTK is described, and implementation issues on the network RTK are outlined.

\subsection{Distance-Dependent Errors}

Distance-dependent errors mean that these errors change from one position to another. DGNSS and RTK positioning technologies are both affected by distance-dependent errors due to the effects of the ionosphere, troposphere, and orbits.

Among different effects, the ionospheric effect is the largest and most variable error source for single-frequency DGNSS and RTK positioning technologies, especially in the case of high ionospheric variability. Figure 1 illustrates how differential ionospheric errors affect positioning solutions. As shown in Figure 1, the ionospheric delay for the reference station is not same as that for the user, and, therefore, the ionospheric error on the user side could not be completely removed by using correction from the reference station. Since ionosphere varies from one location to another location, these ionospheric effects are dependent on both the baseline length and the ionospheric variability. For safety-critical applications, the differential ionospheric delay shown in Figure 1 poses a threat for the integrity performance of the system. Therefore, distance-dependent errors in DGNSS must be accounted for to achieve better accuracy and integrity performance.

\subsection{Modelling Ionosphere in Network RTK}

The integer-ambiguity resolution of carrier phases between reference stations is a prerequisite for the operation of network RTK positioning, and it can be achieved with raw measurements on multiple frequencies and known locations of reference stations [22]. When integer ambiguities on multiple frequencies are correctly fixed, double-differenced residual errors between reference stations can be calculated, mainly including orbit and atmosphere errors. The double-differenced ionospheric delay can then be estimated [20]:

$$
\nabla \Delta I=\frac{f_{2}^{2}}{f_{1}^{2}-f_{2}^{2}}\left(\nabla \Delta \Phi_{1}-\nabla \Delta \Phi_{2}-\lambda_{1} \nabla \Delta N_{1}+\lambda_{2} \nabla \Delta N_{2}\right),
$$

where $\nabla \Delta$ is the double-differencing operation; $I$ is the ionospheric delay on $L 1$ in meters; $f_{1}$ and $f_{2}$ are two GPS frequencies; $\Phi_{1}$ and $\Phi_{2}$ are carrier phases in meters on $L 1$ and $L 2$, respectively; and $N_{1}$ and $N_{2}$ are integer ambiguities in cycles on $L 1$ and $L 2$, respectively. 


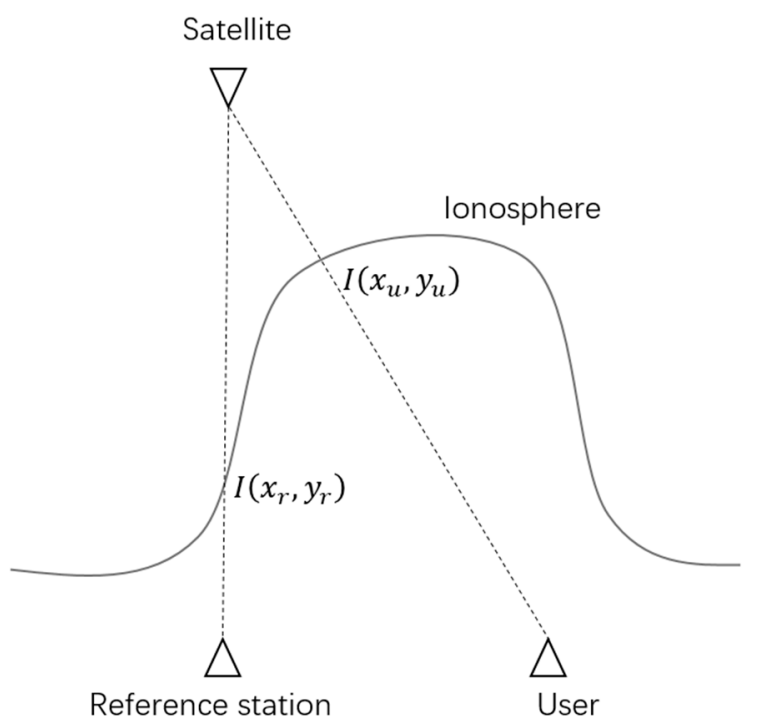

Figure 1. Effects of differential ionospheric delay on differential global navigation satellite system (DGNSS) performance.

To enable error estimation at the user location, the double-differenced ionospheric delay from multiple reference stations is modelled using different methods, e.g., the linear model, the distance-based linear model, and the low-order surface model. Dai et al. [23] compared these different models and found that there were no significant differences between them. As an example, the simple linear model is given as follows.

$$
\nabla \Delta I=a \Delta X+b \Delta Y
$$

where $\Delta X$ and $\Delta Y$ are differences in coordinates in the horizontal plane between master and auxiliary reference stations, and $a$ and $b$ are the model coefficients. Some researchers indicated that the distance-dependent ionosphere error can be modelled at the ionospheric piece point instead of the receiver coordinates. Their difference is very small and can almost be neglected. Therefore, receiver coordinates were used in this paper to model distance-dependent errors.

The model in Equation (2) requires at least three reference stations that are connected to a server. Figure 2 illustrates an example of network RTK. There are five reference stations, denoted by $1,2,3,4$, and $n$, and a user denoted by $u$ in the example. Suppose that the reference satellite is $q$, and the master station is $n$. For a satellite $p$, parameters can usually be computed using the least-squares method.

$$
\left[\begin{array}{l}
a^{p, q} \\
b^{p, q}
\end{array}\right]=\left(A^{T} A\right)^{-1} A^{T}(\nabla \Delta I),
$$

where

$$
A=\left[\begin{array}{cc}
\Delta X_{1, n} & \Delta Y_{1, n} \\
\Delta X_{2, n} & \Delta Y_{2, n} \\
\vdots & \vdots \\
\Delta X_{n-1, n} & \Delta Y_{n-1, n}
\end{array}\right] \text { and } \nabla \Delta I=\left[\begin{array}{c}
\nabla \Delta I_{1, n}^{p, q} \\
\nabla \Delta I_{2, n}^{p, q} \\
\vdots \\
\nabla \Delta I_{n-1, n}^{p, q}
\end{array}\right]
$$

For the user location, the correction can be interpolated on the basis of the following equation:

$$
\nabla \Delta I_{u, n}^{p, q}=\left[\Delta X_{u, n}, \Delta Y_{u, n}\right]\left[\begin{array}{l}
a^{p, q} \\
b^{p, q}
\end{array}\right],
$$

where $u$ is the user receiver. 


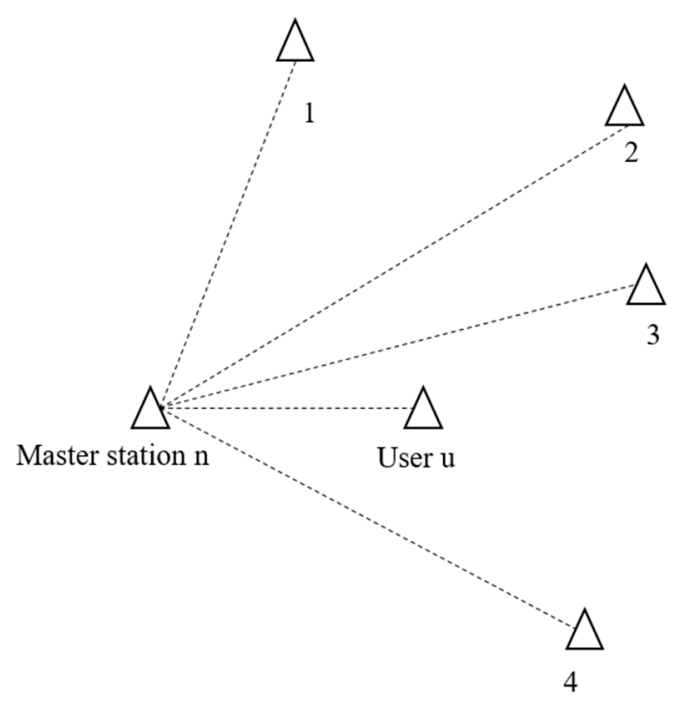

Figure 2. Network real-time kinematic (RTK) principle.

In the market, network RTK can be achieved via different correction technologies, such as master auxiliary concept (MAC), area-correction parameters (in German, Flächenkorrekturparameter, FKP), and virtual reference stations (VRS) [21]. These technologies differ in the means of delivering messages from reference station to rover, and most are supported by leading GNSS receiver manufacturers. The MAC provides the rover with raw measurements from the master reference station and network correction differences via separate messages. Therefore, the rover software of the MAC needs to compute the model parameters and then interpolate the correction at its own location. FKP is another method that provides the rover with raw measurements of the master reference station and the model parameters, accounting for distance-dependent errors via different messages. Therefore, an FKP rover does not need to model the errors, only to interpolate the correction at its own location. VRS is another method that provides virtual measurements from a nonphysical reference station near the rover. If a VRS is adopted to implement network RTK, the interpolation of network corrections is performed in the master reference station, and model parameters are not provided by standard messages.

In both MAC and FKP, the model parameters accounting for distance-dependent errors are available in the rover. In VRS, however, these parameters are not available on the rover side, but they are on the server side.

In this study, the FKP parameters of the Hong Kong network's RTK service were accessed and recorded on 13 March 2021, when solar activity was very low. Figure 3 shows real-time vertical ionospheric gradients on GPS L1 in two directions on 13 March 2021. Although solar activity was not very active in 2021, the largest vertical north-south gradient reached $10 \mathrm{~mm} / \mathrm{km}$, while the largest gradient in the east-west direction was about $5 \mathrm{~mm} / \mathrm{km}$. The vertical ionospheric gradient in the north-south direction was much higher than that in the east-west direction due to the fountain effects in low-latitude areas. The ionospheric gradient peaks ay around 14:00 local time, which was confirmed in our previous studies $[3,9,19]$.

Historical ionospheric gradients were extensively investigated because they pose potential threats to the integrity of the DGNSS or GBAS, which play a critical role in aircraft applications. For the slant gradient, $3 \mathrm{~mm} / \mathrm{km}$ is a conservative value [8]. However, the ionospheric gradient in low-latitude regions was much larger than that in midlatitude areas. As shown in Figure 3, the vertical gradient of ionosphere on L1 frequency in the north-south direction could reach more than $10 \mathrm{~mm} / \mathrm{km}$, even when solar activity was low. In our previous study, it was reported that the ionospheric gradients in low-latitude regions exceeded $50 \mathrm{~mm} / \mathrm{km}$ on most days in 2001, when solar activity was high [9]. 


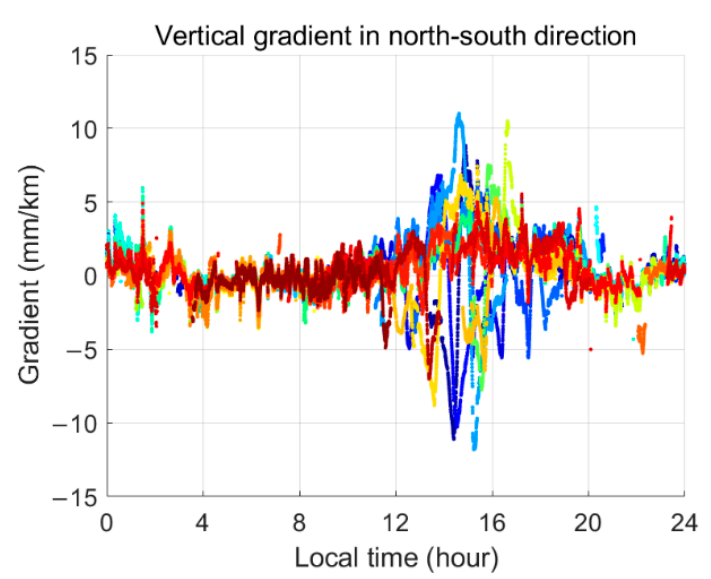

(a)

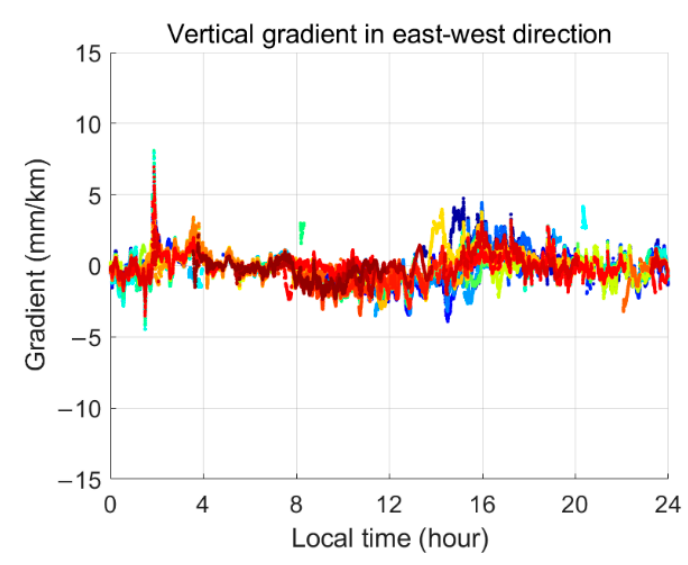

(b)

Figure 3. Vertical ionospheric gradient on global positioning system (GPS) L1 in (a) north-south and (b) east-west directions in Hong Kong on 13 March 2021.

\section{Modifying Code-Based DGNSS with Network RTK Corrections}

The network RTK service has been extensively developed to support high-precision applications. However, the network RTK was mainly developed for carrier-phase measurements. Compared with network RTK, DGNSS was developed for code measurements. The purpose of this study is to improve DGNSS performance through the use of network RTK corrections without modifying the hardware in user receivers. This section presents how to use the network RTK corrections in DGNSS.

In the traditional DGNSS, a rover computes a pseudorange correction (PRC) for satellite $p(p=1,2, \cdots M)$, as follows [24]:

$$
\operatorname{PRC}^{p}(t)=\operatorname{PRC} C^{p}\left(t_{0}\right)+R R C^{p}\left(t_{0}\right)\left[t-t_{0}\right]
$$

where $R R C$ is the change rate of pseudorange correction $P R C$; and $t_{0}$ and $t$ are the generation and application times of the pseudorange correction, respectively. As shown in Equation (5), the traditional DGNSS does not consider the difference in ionospheric delay for a satellite between reference station and rover, i.e., one correction for the distancedependent error.

The double-differenced model of ionospheric delay is provided by the network RTK positioning technology. Suppose that reference satellite $q$ and master reference station $n$ are used in the double-differenced operation. The relationship between the double difference and single difference of ionosphere delay is given in the following equation.

$$
\Delta I_{u, n}^{p}=\Delta I_{u, n}^{q}+\nabla \Delta I_{u, n}^{p, q}
$$

where $u$ is the user receiver, $n$ is the master reference station, $p$ is the reference satellite, and $\nabla \Delta I_{u, n}^{p, q}$ is the interpolated correction at the rover location using network RTK corrections. As shown in Equation (6), the single difference in ionosphere delay for reference satellite is $\Delta I_{u, n}^{q}$, while that for other satellites (denoted as $\left.p\right)$ is $\Delta I_{u, n}^{q}+\left(\Delta X_{u, n}, \Delta Y_{u, n}\right)\left[\begin{array}{l}a^{p, q} \\ b^{p, q}\end{array}\right]$.

Adding Equation (6) to Equation (5) gives the pseudorange correction when the distance-dependent error of ionosphere is considered.

$$
\begin{gathered}
P R C^{p}(t)=P R C^{p}\left(t_{0}\right)+R R C^{p}\left(t_{0}\right)\left[t-t_{0}\right]+\Delta I_{u, n}^{p} \\
=P R C^{p}\left(t_{0}\right)+R R C^{p}\left(t_{0}\right)\left(t-t_{0}\right)+\Delta I_{u, n}^{q}+\left(\Delta X_{u, n}, \Delta Y_{u, n}\right)\left[\begin{array}{l}
a^{p, q} \\
b^{p, q}
\end{array}\right]
\end{gathered}
$$

As shown in Equation (7), the unknown $\left(\Delta I_{u, n}^{q}\right)$ is used to compute the single difference in ionospheric delay for other satellites $\left(\Delta I_{u, n}^{p}\right)$. Fortunately, this unknown is a common 
term in all satellite corrections. That is, this parameter affects receiver clock estimation, while it does not affect position estimation. In the proposed DGNSS, common unknown $\Delta I_{u, n}^{q}$ is ignored. When the single difference in ionosphere delay for the reference satellite $\left(\Delta I_{u, n}^{q}\right)$ is disregarded, the pseudorange correction is given as follows:

$$
P R C^{p}(t)=P R C^{p}\left(t_{0}\right)+R R C^{p}\left(t_{0}\right)\left(t-t_{0}\right)+\left(\Delta X_{u, n}, \Delta Y_{u, n}\right)\left[\begin{array}{l}
a^{p, q} \\
b^{p, q}
\end{array}\right] .
$$

In the modified DGNSS, the rover uses Equation (8) to compute the pseudorange correction.

Figure 4 shows the flowchart of the rover processing. First, parameters from the network RTK services are decoded. The spatial correction is estimated at the rover location as the product of the coordinate difference $\left(\Delta X_{u, n}, \Delta Y_{u, n}\right)$ and model coefficients $\left(a^{p, q}, b^{p, q}\right)$ from the network RTK. The spatial correction is then added to the pseudorange correction of the traditional DGNSS.

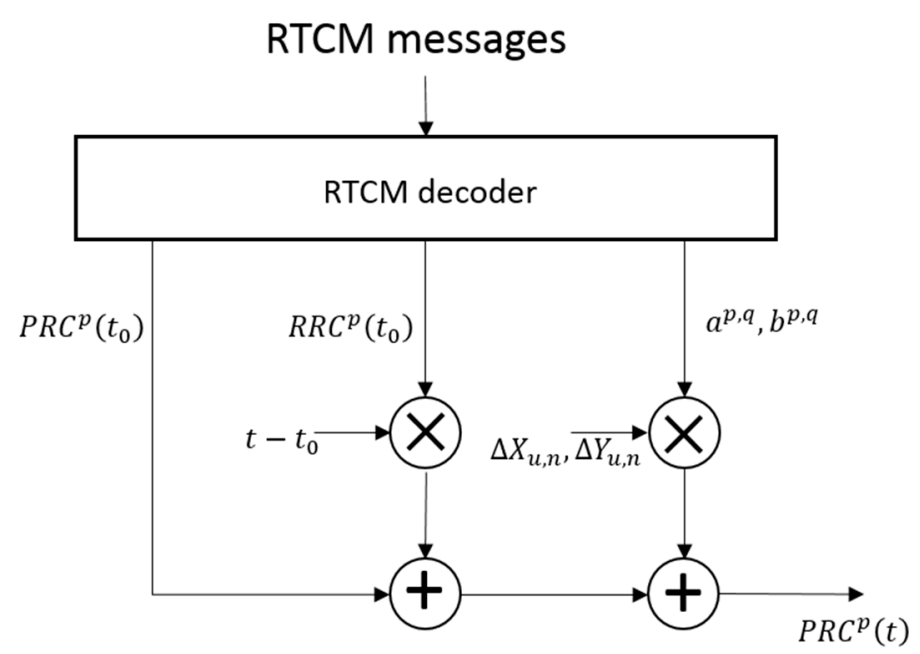

Figure 4. Flowchart for calculating pseudorange correction (PRC) in rover software of modified differential GNSS (DGNSS).

\section{Evaluation of Modified DGNSS}

Located from $22^{\circ} 08^{\prime} \mathrm{N}$ to $22^{\circ} 35^{\prime} \mathrm{N}$ latitude, Hong Kong is characterized by large ionospheric anomalies. The maximal daily ionospheric gradient in solar maximum years varies from 20 to $100 \mathrm{~mm} / \mathrm{km}$, depending on season and local time [9].

The Hong Kong Satellite Positioning Reference Station Network (SatRef) provides both a real-time network RTK service and historical data. In this study, the performance of the proposed DGNSS was evaluated using 2001 data, when solar activity was highest in the past two solar cycles. GNSS receiver independent exchange format (RINEX) data in Hong Kong are provided on the following website: https:/ / www.geodetic.gov.hk/en/ geod_services.htm (accessed on 23 March 2021). As shown in Table 1, two days of GNSS data from the Hong Kong CORS network were selected and processed for the evaluation: one was 4 March 2001, when the ionosphere was quiet, and the other was 6 November 2001, when a geomagnetic storm happened. Kp and Dst indices are also shown to represent the ionospheric condition for each dataset. We used two datasets to represent quiet and disturbed conditions in the low-latitude regions, respectively.

Five reference stations marked with circles in Figure 5 (HKFN, HKKT, HKLT, HKSL, HKST) were selected to provide the network RTK service. The network RTK corrections were then applied in DGNSS between HKKT and KKY, which were selected as the reference station and rover, respectively. The baseline length between HKKT and HKKY was $17.9 \mathrm{~km}$. All of these reference stations are equipped with professional Leica receivers. 
Table 1. Details of testing observation data.

\begin{tabular}{ccccc}
\hline Date & Kp & Dst & $\begin{array}{c}\text { Ionospheric } \\
\text { Condition }\end{array}$ & $\begin{array}{c}\text { Reference } \\
\text { Stations }\end{array}$ \\
\hline 4 March 2001 & 3 & -31 & Quiet & HKFN, HKLT, \\
& & & HKKT, HKST, \\
HKSL, HKKY
\end{tabular}

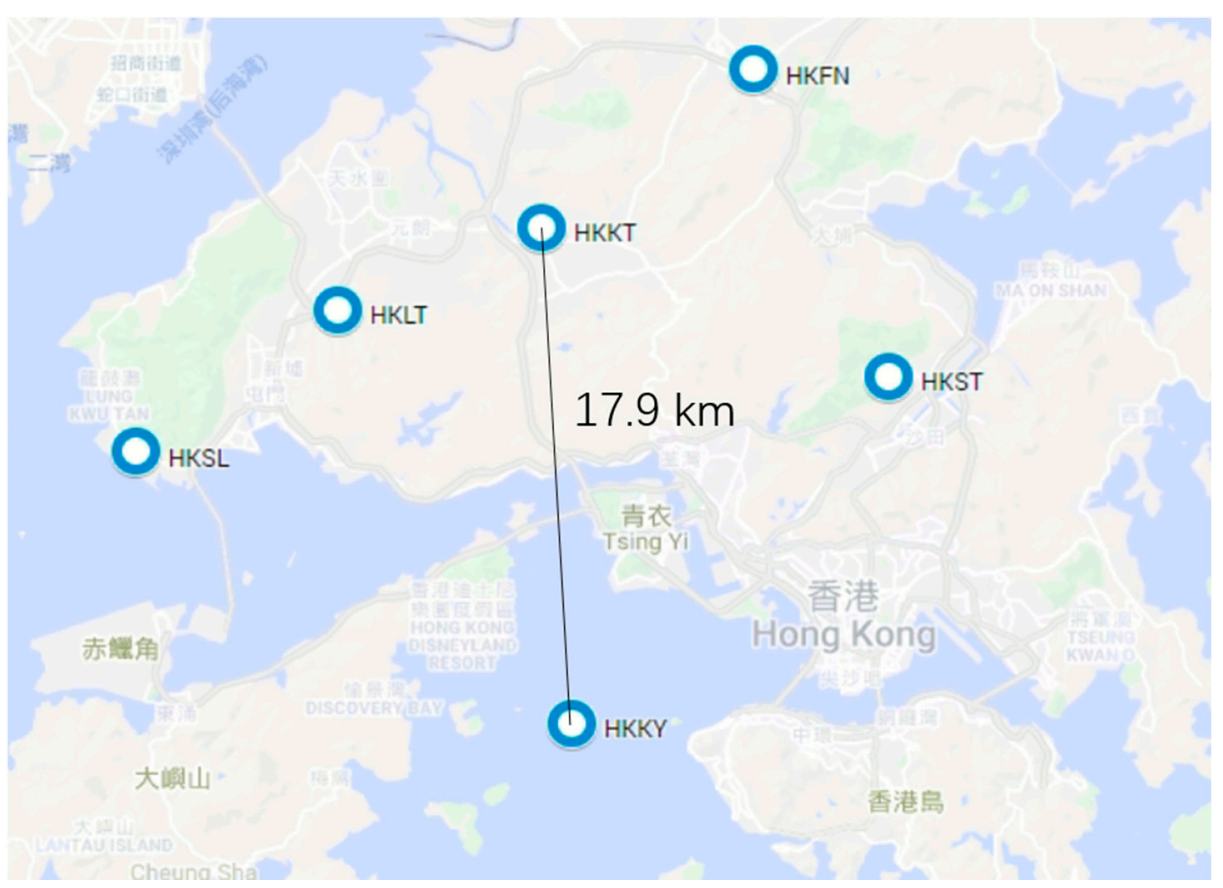

Figure 5. Distribution of selected reference stations in Hong Kong.

Dual frequency GNSS data from five reference stations were firstly post processed with a network RTK software. In the software, the double-differenced ionospheric delay was calculated after ambiguities on two frequencies had been resolved, as shown in Equation (1). With the known locations of reference stations, the double-differenced ionospheric delay was modelled using a linear function of coordinate differences in the horizontal plane between master and auxiliary reference stations, as shown in Equation (2). The parameters of the model include the ionospheric the east-west and north-south gradients.

The dual-frequency carrier phase measurements are available from the reference station HKKT and KKKY. This enables us to evaluate the accuracy of the differential ionospheric delay. The double-differenced ionospheric delay predicted from the linear model was compared with that calculated from the dual-frequency carrier phase measurements. It was found that the RMS of their differences was $0.04 \mathrm{~m}$ on 4 March 2001 and $0.05 \mathrm{~m}$ on 9 November 2001. It can be seen that the accuracy of the ionospheric linear model used in this study is sufficient for its application in DGNSS.

Figures 6 and 7 show the ionospheric gradients on 4 March 2001 and 6 November 2001. Various satellites are distinguished by different colors. Compared with gradients in the eastwest direction (Figures $6 a$ and $7 \mathrm{a}$ ), gradients in the north-south direction (Figures $6 \mathrm{~b}$ and $7 \mathrm{~b}$ ) were much larger. The larger gradients in the north-south direction resulted from the equatorial anomaly, namely, the "fountain effect", which depends on location, solar activity, season, and local time. During active solar years, such as 2001-2003, the large spatial gradient caused by the equatorial anomaly can be frequently observed in Hong Kong. 
Compared with the ionospheric gradients in Figure 6, the ionospheric gradients in Figure 7 is not significantly large. We also investigated the non-differenced ionospheric delay derived from dual-frequency GNSS data in HKKY station on two days. It was observed that the non-differenced ionospheric delay on 6 November 2001 was much larger than that on 4 March 2001. Therefore, the geomagnetic storm on 6 November 2001 caused significant changes in the non-differenced ionospheric delay, instead of abnormal ionospheric gradients in Hong Kong. This can be caused by the fact that the ionospheric gradient, shown in Figure 7, covers an area much larger than the test area (tens of kilometers).

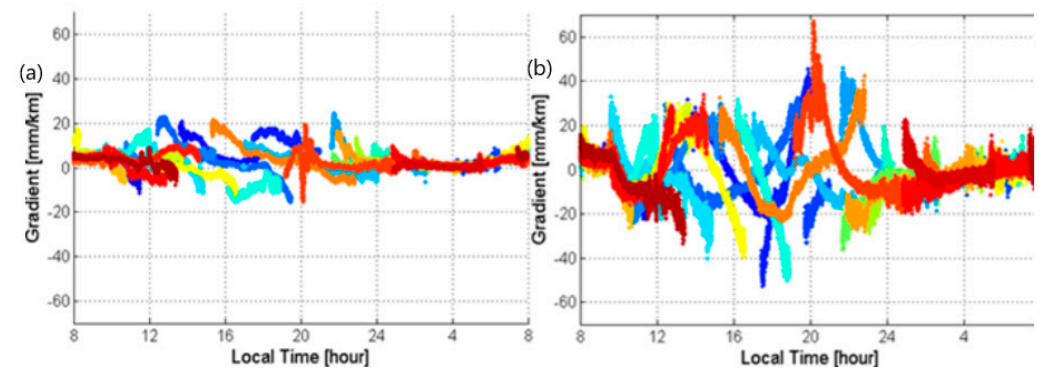

Figure 6. The east-west gradient (a) and the north-south gradient (b) for the ionospheric delay on 4 March 2001.
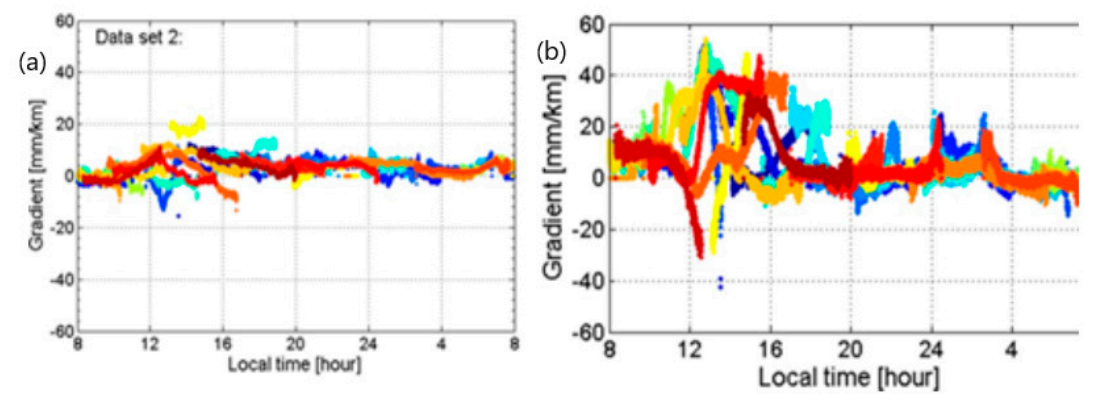

Figure 7. East-west gradient (a) and north-south gradient (b) for the ionospheric delay on 6 November 2001 [9].

One the user side of the proposed DGNSS, the additional ionospheric correction for each satellite is estimated using the linear model, as shown in Equation (4). The new pseudorange correction is obtained by adding the additional ionospheric correction to the original pseudorange correction generated from the reference station HKKT, as shown in Equation (8). The new pseudorange correction of the proposed DGNSS is then applied in receivers to solve for the user position in the same way as normal DGNSS.

Analysis was then carried out by comparing the positioning errors of two solutions: the conventional DGNSS, and the DGNSS modified by using network RTK corrections. To evaluate the validity of the proposed DGNSS, the conventional DGNSS solution was first performed for the HKKT-HKKY baseline. Then, the same baseline (HKKT-HKKY) was processed by DGNSS with the use of the network RTK corrections. Positioning errors were obtained by comparing the solution with the known locations of reference stations that had been precisely surveyed. In Figures 6 and 7, gradients changed with the local time, and the period from 11:00 to 18:00 local time was chosen for further investigation because gradients were much larger during this period.

Figures 8 and 9 show the positioning errors of the conventional DGNSS without using the network RTK corrections as the green curve, and that of the solution using network RTK corrections as the red curve. As shown, performance was improved when network RTK corrections had been applied during the period from 11:00 to 18:00 local time. In both figures, the panel (a) shows the horizontal positioning errors, and the panel (b) shows 
the vertical positioning errors. It can be seen from Figures 8 and 9 that there a significant improvement in the horizontal positioning errors.
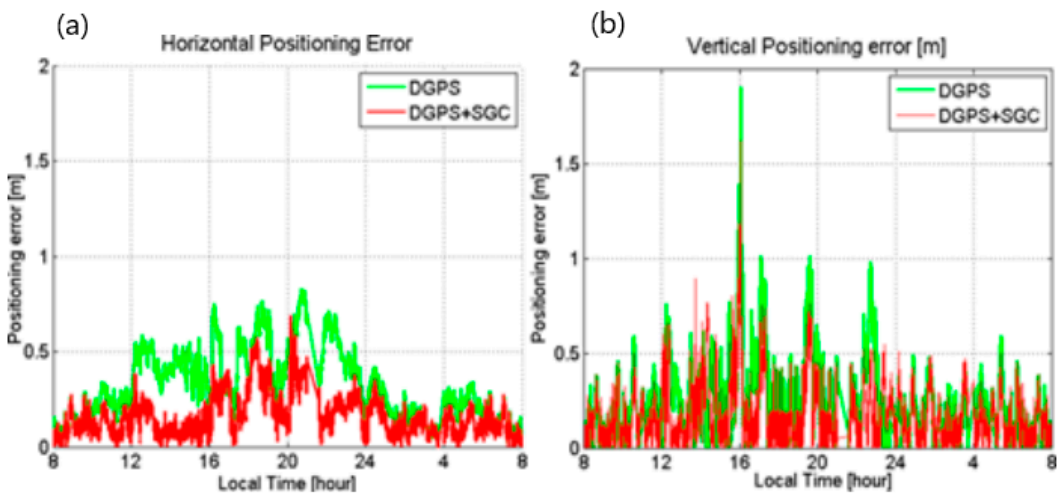

Figure 8. Horizontal (a) and vertical (b) positioning DGNSS results (HKKT-HKKY) on 4 March 2001.
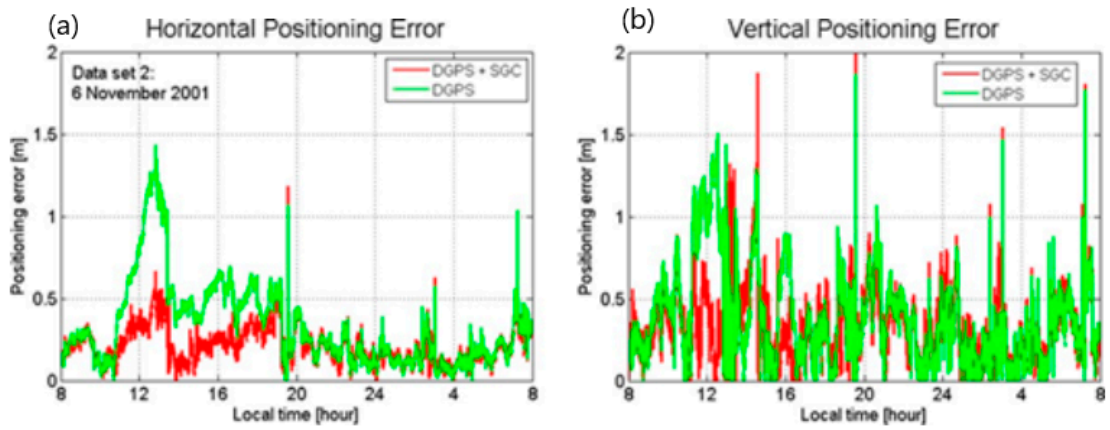

Figure 9. Horizontal (a) and vertical (b) positioning DGNSS results (HKKT-HKKY) on 6 November $2001[9]$.

For the $17.9 \mathrm{~km}$ baseline length, the RMS of the horizontal positioning error during the period was improved from 0.52 to $0.24 \mathrm{~m}$ on 4 March 2001, and from 0.72 to $0.27 \mathrm{~m}$ on 6 November 2001, as shown in Table 2. The horizontal positioning error could be improved by $53 \%$ and $62 \%$, respectively, by using network RTK corrections. Thus, network RTK corrections can reduce positioning error in the low-latitude areas, especially during the daytime.

Table 2. Improvements in DGNSS positioning error by using network RTK corrections.

\begin{tabular}{ccccccc}
\hline \multirow{2}{*}{ Date } & \multicolumn{2}{c}{ Horizontal Positioning Error (Meter) } & \multicolumn{2}{c}{ Vertical Positioning Error (Meter) } \\
\cline { 2 - 7 } & DGNSS & $\begin{array}{c}\text { Modified } \\
\text { DGNSS }\end{array}$ & $\begin{array}{c}\text { Improvement } \\
\mathbf{( \% )}\end{array}$ & DGNSS & $\begin{array}{c}\text { Modified } \\
\text { DGNSS }\end{array}$ & $\begin{array}{c}\text { Improvement } \\
(\%)\end{array}$ \\
\hline 4 March 2001 & 0.52 & 0.24 & 53 & 0.44 & 0.38 & 14 \\
\hline 6 November 2001 & 0.72 & 0.27 & 62 & 0.73 & 0.49 & 32 \\
\hline
\end{tabular}

\section{Conclusions}

DGNSS performance can be degraded by temporal and spatial decorrelation errors. Temporal decorrelation errors can be mitigated by using the RRC, while spatial decorrelation is not corrected in the local-area DGNSS. When the SA was switched off, spatial decorrelation became the most prominent error source. To reduce performance degradation with distance from the reference station, a modified DGNSS using network RTK corrections was proposed to mitigate the spatial decorrelation error.

This study evaluated the performance of the proposed DGNSS with the use of network RTK corrections by processing historical data. GNSS data from two days in 2001 (the solar 
maximum year), recorded by the Hong Kong CORS network, were processed for the assessment: 4 March, when the ionosphere had been quiet, and 6 November, when a geomagnetic storm had happened. By applying network RTK corrections, the RMS of the horizontal positioning error was improved from 0.52 to $0.24 \mathrm{~m}$ for the first dataset, and 0.72 to $0.27 \mathrm{~m}$ for the second dataset. In two tests, the horizontal positioning error was improved by $53 \%$ and $62 \%$, respectively, when using network RTK corrections. It should be noted that the behavior of the ionosphere and troposphere is unpredictable and can vary a lot. The two data sets used in this study are small samples, and the achievable accuracy of the proposed DGNSS can be better or weaker for some other samples. For example, the accuracy of DGNSS-FKP, performed in high latitude, was improved by a maximum of $40 \%$ [6].

The proposed DGNSS could be achieved in real time by using network RTK technologies in the market. When network RTK positioning is implemented using FKP technology, gradient parameters are readily broadcast via Radio Technical Commission for Maritime Services (RTCM) messages, and the rover can use parameters to estimate spatial decorrelation errors. When network RTK positioning is implemented using MAC technology, distance-dependent errors should first be modelled in the rover, and corrections for the spatial decorrelation errors can then be estimated. The standard VRS technology itself does not provide model parameters to rovers. However, the model parameters are available on the server side. The modified DGNSS could be achieved via, for example, VRS, in which pseudorange corrections are provided on the server side with the approximate position of the rover.

Author Contributions: Conceptualization, D.W. and S.J.; methodology, D.W. and Z.L.; software, D.W.; validation, W.C. and Y.L.; formal analysis, Y.L.; investigation, W.C.; resources, W.C.; data curation, S.J.; writing-original-draft preparation, D.W.; writing-review and editing, D.W. and Y.L.; visualization, Z.L.; supervision, W.C.; project administration, W.C.; funding acquisition, W.C. All authors have read and agreed to the published version of the manuscript.

Funding: This research was funded by the National Key Research and Development Program of China (grant numbers 2016YFB0501803), the Shenzhen Science and Technology Innovation Commission (grant number JCYJ20170818104822282), and the Hong Kong Research Grants Council (RGC; grant number 152223/18E).

Institutional Review Board Statement: Not applicable.

Informed Consent Statement: Not applicable.

Data Availability Statement: The GNSS reference data in history and real-time network RTK corrections are available from Hong Kong reference stations provided by Hong Kong Lands department (https:/ / www.geodetic.gov.hk/en/geod_services.htm accessed on 23 March 2021).

Conflicts of Interest: The authors declare no conflict of interest.

\section{References}

1. Weng, D.; Gan, X.; Chen, W.; Ji, S. A new DGNSS positioning infrastructure for android smartphones. Sensors 2020, 20 , 487. [CrossRef] [PubMed]

2. Park, B.; Kim, J.; Kee, C.; Cleveland, A.; Parsons, M.; Wolfe, D. RRC Unnecessary for DGPS messages. IEEE Trans. Aerosp. Electron. Syst. 2006, 42, 1149-1160. [CrossRef]

3. Skone, S. Limitations in DGPS positioning accuracies at low latitudes during solar maximum. Geophys. Res. Lett. 2002, 29, 1439. [CrossRef]

4. Kim, S.; Park, K. Improving DGPS accuracy by considering the correlation of pseudorange correction and satellite elevation angle. J. Navig. 2017, 70, 1267-1275. [CrossRef]

5. Lee, J.; Seo, J.; Park, Y.; Pullen, S.; Enge, P. Ionospheric threat mitigation by geometry screening in Ground-Based Augmentation Systems. J. Aircr. 2011, 48, 1422-1433. [CrossRef]

6. Kim, J.; Song, J.; No, H.; Han, D.; Kim, D.; Park, B.; Kee, C. Accuracy Improvement of DGPS for Low-Cost Single-Frequency Receiver Using Modified Flächen Korrektur Parameter Correction. ISPRS Int. J. GeoInf. 2017, 6, 222. [CrossRef]

7. Minimum Aviation Systems Performance Standards for Local Area Augmentation System (LAAS); RTCA DO-245A; RTCA Inc.: Washington, WA, USA, 2004. 
8. Datta-Barua, S.; Lee, J.; Pullen, S.; Luo, M.; Ene, A.; Qiu, D.; Zhang, G.; Enge, P. Ionospheric Threat Parameterization for Local Area Global-Positioning-System-Based Aircraft Landing Systems. J. Aircr. 2010, 47, 1141-1151. [CrossRef]

9. Weng, D.; Ji, S.; Chen, W.; Li, Z.; Xu, Y.; Ye, L. Assessing and mitigating the effects of the ionospheric variability on DGPS. GPS Solut. 2015, 19, 107. [CrossRef]

10. Weng, D.; Chen, W. SBAS enhancement using an independent monitor station in a local area. GPS Solut. 2019, 23, 1-9. [CrossRef]

11. Odijk, D.; van der Marel, H.; Song, I. Precise GPS positioning by applying ionospheric corrections from an active control network. GPS Solut. 2000, 3, 49-57. [CrossRef]

12. Bakuła, M. Network code DGPS positioning and reliable estimation of position accuracy. Surv. Rev. 2010, 42, 82-91. [CrossRef]

13. Przestrzelski, P.; Bakuła, M.; Galas, R. The integrated use of GPS/GLONASS observations in network code differential positioning. GPS Solut. 2017, 21, 627. [CrossRef]

14. Raman, S.; Garin, L. Performance Evaluation of Global Differential GPS (GDGPS) for Single Frequency C/A Code Receivers. In Proceedings of the 18th International Technical Meeting of the Satellite Division of The Institute of Navigation (ION GNSS 2005), Long Beach, CA, USA, 13-16 September 2005; pp. 1465-1469.

15. Kee, C.; Yun, D. Extending Coverage of DGPS by Considering Atmospheric Models and Corrections. J. Navig. 2002, 55, 305-322. [CrossRef]

16. Li, B.; Shen, Y.; Feng, Y.; Gao, W.; Yang, L. GNSS ambiguity resolution with controllable failure rate for long baseline network RTK. J. Geod. 2014, 88, 99-112. [CrossRef]

17. Rizos, C.; Han, S. Reference station network based RTK systems-Concepts and progress. Wuhan Univ. J. Nat. Sci. 2003, 8, 566-574. [CrossRef]

18. Wanninger, L. Improved AR by regional differential modeling of the ionosphere. In Proceedings of the 8th International technical meeting of the Satellite Division of the US Institute of Navigation, Palm Springs, CA, USA, 12-15 September 1995 ; pp. 55-62.

19. Tang, W.; Jin, L.; Cui, J.; Shi, C.; Zhang, Y. GNSS Network RTK regional ionospheric modelling studies and performance analysis. J. Navig. 2016, 69, 211-224. [CrossRef]

20. Cui, J.; Tang, W.; Jin, L.; Deng, C.; Zou, X.; Gu, S. An improved ionosphere interpolation algorithm for network RTK in low-latitude regions. GPS Solut. 2018, 22, 109. [CrossRef]

21. Janssen, V. A Comparison of the VRS and MAC principles for network RTK. In Proceedings of the IGNSS Symposium, Surfers Paradise, Australia, 1-3 December 2009.

22. Hu, G.; Abbey, D.A.; Castleden, N.; Featherstone, W.E.; Earls, C.; Ovstedal, O.; Weihing, D. An approach for instantaneous ambiguity resolution for medium to long-range multiple reference station networks. GPS Solut. 2005, 9, 1-11. [CrossRef]

23. Dai, L.; Han, S.; Wang, J.; Rizos, C. Comparison of Interpolation Algorithms in NetworkBased GPS Techniques. J. Inst. Navig. 2004, 50, 277-293. [CrossRef]

24. Radio Technical Commission for Marine Services. RTCM Recommended Standards for Differential GNSS (Global Navigation Satellite Systems) Service, version. 2.3; RTCM: Washington, WA, USA, 2001. 